\title{
LENSING LIMITS ON THE COSMOLOGICAL CONSTANT
}

\author{
HANS-WALTER RIX \\ Max-Planck-Institut für Astrophysik \\ Postfach 1523, D-85740 Garching, Germany
}

\begin{abstract}
The statistics of gravitationally lensed quasars requires that $\lambda<0.7$ at $90 \%$ confidence. This limit is weakly affected by the evolution of galaxies through merging. If $\mathrm{E} / \mathrm{S} 0$ galaxies have much higher dust opacities at redshifts of 0.5-1.0 than they do locally, these limits can be evaded.
\end{abstract}

\section{Why care about $\lambda$ ?}

After spending much of its life in the corner-cabinet for "unorthodox cosmologies", the cosmological constant, $\lambda$ (or $\Omega_{\lambda}$ ) has become socially acceptable in the last years (see Caroll, Press and Turner (1991), for a review). Cosmologists have reminded themselves that the equation governing the expansion of the universe

$$
\mathrm{H}^{2} \equiv\left(\frac{\dot{R}}{R}\right)^{2}=\frac{8 \pi G}{3} \rho_{\text {matter }}-\frac{k}{R^{2}}+\frac{\Lambda}{3}
$$

indeed may contain the constant $\Lambda \neq 0^{1}$. The aesthetic arguments for expecting $\lambda=0$ are weak and therefore the value of $\lambda$ should be determined empirically. However, this realization in itself was not sufficient to prompt widespread interest in measuring the actual value of $\lambda$. This interest has been sparked largely by the persistent conflict (see, e.g. Freedman et al.1995), between the age determination of globular clusters ( $~ 15 \mathrm{Gyrs})$ and the estimates of the Hubble constant $\left(\approx 65 \mathrm{~km} \mathrm{~s}^{-1} \mathrm{Mpc}^{-1}\right)$, which lead to a universe younger than some globular clusters for cosmologies with $\lambda=0$. Yet, such an age conflict would not exist in a flat universe with $\Omega_{\text {matter }}=0.3$ and $\lambda=0.7$.

\footnotetext{
${ }^{1}$ This constant is usually expressed as its current epoch contribution to $\Omega: \lambda \equiv \Omega_{\lambda} \equiv$ $\Lambda /\left(2 H_{0}^{2}\right)$
} 


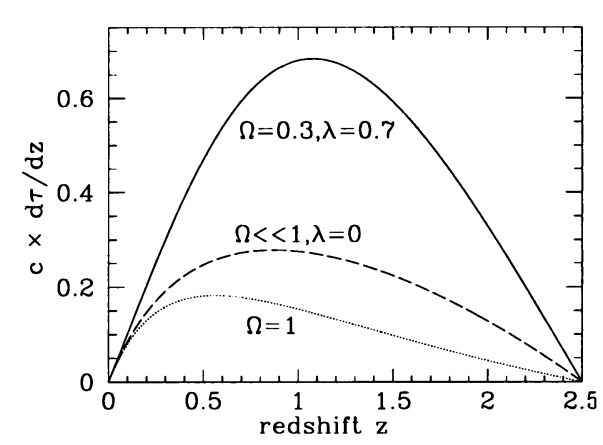

Figure 1. The relative optical depth to lensing in different cosmologies. All other things equal, many more lenses are expected in $\lambda$-dominated cosmologies

\section{2. $\lambda$ determinations from lensing statistics}

It was suggested about six years ago (Fukugita et al.1990, Turner 1990) that the statistics of multiply lensed objects, such as QSOs, might provide a useful test for $\lambda$-dominated cosmologies. The differential optical depth for multiple imaging, $d \tau / d z$, due to a lens population of local space density $n_{l}$, can be written as the probability of intercepting a target of area unity, $d P / d z$, times the cross-section for image splitting, $A_{c r i t}$. These quantities are given by $d P / d z=n_{l}(z=0)(1+z)^{3} d l / d z$ and $A_{c r i t}=f_{l}(\sigma) D_{O L} D_{L S} / D_{O S}$. The factor $f_{l}(\sigma)$ depends on the mass profile of the lens (mostly the velocity dispersion $\sigma$ ) and it is for now assumed that the lens population does not evolve in co-moving coordinates. The optical depth can be written as

$$
\frac{d \tau}{d z}=n_{l}(0) f_{l}(\sigma) \times(1+z)^{3} \frac{d l}{d z} \frac{D_{O L} D_{L S}}{D_{O S}} .
$$

While the first two factors only depend on the properties of the lenses, the last three factors only depend on the geometry of the universe. These geometrical factors are plotted in Figure 1 for a source population at $\langle z\rangle=$ 2.5 for three cosmologies: (a) $\Omega_{\text {matter }} \ll 1, \lambda=0$, (b) $\Omega_{\text {matter }}=1, \lambda=0$ and (c) $\Omega_{\text {matter }}=0.3, \lambda=0.7$. The optical depth to lensing for a given lens population is $4-5$ times higher in a flat universe with $\lambda=0.7$ than with $\lambda=0$. Therefore, with a statistically well defined sample of sources, e.g. distant QSOs, and with an independently characterized population of lenses, one can hope to constrain $\lambda$ from the statistics (mostly the frequency and separation distribution ${ }^{2}$ ) of multiple images.

\footnotetext{
${ }^{2}$ But see Kochanek (1992) for a different statistical $\lambda$ test, employing the distribution of lens-redshifts. In general, all observational constraints can and should be included in a likelihood analysis
} 


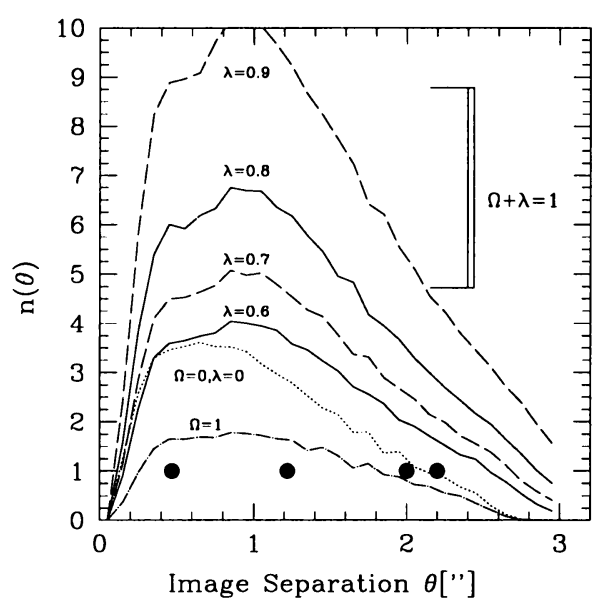

Figure 2. Lens Models for the HST Snapshot-Survey. Shown is the number of expected lenses per separation interval, $\mathrm{n}(\theta)$, vs. the separation $\theta$. The solid dots indicate the separations of the observed lenses.

\section{Application to the HST Snapshot Survey}

The practical application of this cosmological test became possible with the advent of a suitable and statistically well defined sample of distant sources $^{3}$, the HST Gravitational Lens Snapshot survey (Maoz et al.1993, and references therein). About 500 luminous QSOs with redshifts $>1$ were surveyed for multiple images, down to separations of $\Delta \theta \gtrsim 0.1$ " for magnitude differences of $\Delta m \lesssim 2.5$. This survey found only one new lens at 0.47 " separation (Bahcall et al.1992) in addition to four previously known lenses.

This data set was modeled independently by Maoz and Rix (1993) and Kochanek (1993). In these models the relevant lens population was taken to be the locally observed elliptical galaxies; these were assumed to have constant co-moving density and fixed mass profiles. The mass normalization of the lenses (with dark halos) can be determined both by a combination of independent stellar-dynamical constraints and by the separations of the observed multiple images. With a conventional cosmology (either $\Omega=1$ or $\Omega \ll 1, \lambda=0$ ), such empirical, non-evolving lens models, can explain the observed abundance and separation distribution $n(\theta)$, once the amplification bias and the survey detection limits are accounted for (see Maoz \& Rix 1993 and Kochanek 1993 for details). In contrast, for a $\lambda$-dominated cosmology these models predict many more lenses than observed. Formally, Maoz and Rix (1993) found $\lambda<0.7$ at $90 \%$ confidence (Figure 2). Kochanek (1993) found $\lambda \lesssim 0.8$ with less constrained lens models; the formal limits of

\footnotetext{
${ }^{3}$ The absence of statistically complete information about the source redshifts in radio surveys complicate the lensing analysis. Here we focus on optically selected samples, despite their drawbacks (Section 4.2)
} 
his likelihood analysis have improved to $\lambda<0.55(2 \sigma)$ with the inclusion of further data (Kochanek 1995). For determining limits on $\lambda$ it is conservative to account only for the well understood lens population of elliptical galaxies; if other objects (groups, dark potential wells, etc..) also contribute, the limits on $\lambda$ will become tighter.

\section{Loop-Holes and Caveats}

These limits on the cosmological constant were (and had to be) derived in the context of a model, predicting the abundance and separation of multiple images. For this result to be useful, it must not be very sensitive to the model assumptions. In the following we discuss the two most critical assumptions that enter the models.

\subsection{EVOLUTION OF THE LENS POPULATION}

The models by Maoz and Rix (1993) and Kochanek (1993) assume that the locally observed lens population is uniformly (in co-moving co-ordinates) sprinkled throughout the universe. In this respect the models ignore the vast body of theoretical prediction and observational evidence that galaxies evolve with cosmological epoch. It may seem hopeless to implement our fragmentary knowledge of galaxy evolution into such lensing models. Can they make meaningful predictions nonetheless? For several reasons these models may indeed place robust upper limits on $\lambda$ : First, we only have to consider the evolution of the assumed lens population, elliptical galaxies with $l \gtrsim 0.3 L_{*}$ and only to redshifts $\approx 1$; this is a small and relatively well understood subset of all galaxies. The observed small scatter in the colormagnitude relation for ellipticals (e.g. Bower et al.1992), and the evolution of the fundamental plane with redshift (e.g. Franx 1993) indicate that the bulk of stars in these galaxies has formed at redshifts beyond unity. However, these galaxies may still evolve by merging. Assuming simple merging histories, Mao and Kochanek (1994) and Rix et al.(1994) addressed the effect of such evolution on the lensing statistics. Both N-body simulations and the existence of the "Fundamental Plane" indicate that the merger of two progenitor galaxies with characteristic dispersion, $\sigma_{i}$, leads to a product with $\sigma_{f}=2^{1 / 4} \sigma_{i}$. Since for an isothermal sphere the cross section increases as $\sigma^{4}$, the total cross-section is approximately conserved under merging: $2 A_{\text {crit }}^{\text {init }} \approx A_{\text {crit }}^{\text {final }}$. As galaxies are "split-up" into smaller pieces, going back in time, the observable distribution of image splittings becomes skewed towards smaller separations. Quantitatively, Rix et al.(1994) found that none of the merging scenarios explored resulted in a "softer" upper limit on $\lambda$ than the no-evolution model. 


\subsection{THE ABUNDANCE OF DUSTY LENSES}

Multiply imaged QSOs are found in very special directions, namely where their light passes through the inner parts (a few $k p c$ ) of a galaxy. During this passage the QSO light may become partially extincted by dust in the galaxy. This would lead to the under-representation of multiple images in a magnitude limited sample, which in turn may permit a dominant $\lambda$ to explain the observed lensing frequency. This is indeed the most serious concern in the modeling procedure, as a good number of radio-selected lens-system show indications of severe reddening (Lawrence et al.1994). However, at this point in time it is not yet conclusively settled in which fraction of these "red radio lenses" the reddening occurs at the redshift of the lens (as opposed to the source).

There is observational evidence that the optical depth due to dust in present-day elliptical is small $(\tau<<1)$ at the impact parameters of lensed images (a few kpc). The dust content of elliptical has been studied extensively through its IR emission (see Goudfrooij (1995) and references therein); these investigations suggest a smoothly distributed dust component, but its optical depth at $R_{e f f}$ is only a few hundredth. A more direct argument comes from the photometry of superimposed cluster ellipticals (Lauer 1988): assume that the foreground galaxy in the superimposed pair contained dust, $\tau_{f}\left(R_{f}\right)$, with a radial profile comparable to the stars', $I_{f}\left(R_{f}\right)$. The centers of the two galaxies are separated by $D$. Now consider the intensity, $I_{1}$, at a distances $R_{b}(<D)$ and $I_{2}$ at $-R_{b}$ from the center of the background galaxy. The intensities are given by

$$
\begin{array}{lc}
I_{1}= & I_{f}\left(R_{f}\right)+I_{b}\left(R_{b}\right) \exp \left(-\tau\left(R_{f}\right)\right) \\
I_{2}= & I_{f}\left(R_{f}+2 D\right)+I_{b}\left(R_{b}\right) \exp \left(-\tau\left(R_{f}+2 D\right)\right) \approx I_{b}\left(R_{b}\right),
\end{array}
$$

where the exponential factors are unity in the dust free case. Lauer found that in the majority of cases the light distributions could be decomposed into two sets of nearly concentric ellipses, as expected for the superposition of symmetric, dust-free galaxies. Compared to transparent galaxies, the intensity of the background galaxy at $\pm R_{b}$ in the dusty case would differ by $\Delta I_{b}\left(R_{b}\right)=I_{b}\left(R_{b}\right)\left[1-\exp \left(-\tau\left(R_{f}\right)\right)\right]$ between the two opposite, but equidistant, point from the center. When fitting isophotes this would lead to a center shift, $\Delta R_{b}$, of $\Delta R_{b} / R_{b} \approx \tau\left(R_{f}\right)\left(\partial \ln I_{b} / \partial \ln R_{b}\right)^{-1}$ for $\tau\left(R_{f}\right) \ll 1$, where $\partial \ln I_{b} / \partial \ln R_{b} \sim 1-3$ is the logarithmic luminosity gradient of the background galaxy. Quantitatively, the upper limits on $\Delta R_{b}$ in Lauer's data indicate $\tau\left(R_{f}\right)<0.04$, with $R_{f}$ of order $k p c$. These data indicate that at least in local ellipticals, dust extinction is unimportant at the level relevant to lensing statistics. 


\section{Future Prospects}

On the face of it, the statistics of gravitational lensing provide a limit on $\lambda$ that makes the cosmological constant almost uninteresting for solving the "age problem" and other outstanding cosmological issues. What can be done in the near future to tighten these limits and to make them less model dependent? On the one hand, we can expect the completion of the MIT-Greenbank survey and the ongoing CLASS survey. These surveys will greatly increase the amount of radio data for lensing statistics. Similarly, the Sloan-Survey will increase tremendously the database of optically selected QSO. Maybe even more important, it should be possible to close some of the loop-holes in the lens modeling: (1) Detailed modeling of a few individual lenses (see Kochanek in this volume) will improve our understanding of how the lensing properties of (E/S0) galaxies, are related to their photometric and stellar kinematic properties; in turn this will constrain better the statistical lensing properties of the photometrically defined lens population. (2) Direct observations with HST will also lead to rapid improvement in our understanding of the galaxy evolution for $0<z<1$. (3) HST observations will further allow us to study the dust content of elliptical galaxies at intermediate redshifts. These observations may lay to rest the of the most worrisome systematic uncertainty in the modeling, preferential dust extinction of multiply imaged objects. Taken together, these arguments give reason to be optimistic that within a few years lensing statistics will settle whether $\lambda$ is cosmologically important, or not.

\section{References}

Bahcall, J., et al., 1992, ApJL, 392, L1

Bower, R., Lucey, J. \& Ellis, R., 1992, MNRAS, 254, 601

Carroll, S., Press, W. \& Turner, E., 1992, ARAA, 30, 499

Franx, M., 1993, ApJL, 407, L5

Freedman, W., et al., 1995, Nature, 371, 757

Fukugita, M., Futamase, T. \& Kasai, M., 1990, MNRAS, 246, 25p

Goudfrooij, P., 1995, PASP, 107, 502

Kochanek, C.S., 1992, ApJ, 384, 1

Kochanek, C.S., 1993, ApJ, 419, 12

Kochanek, C.S., 1995, ApJ, submitted.

Lauer, T., 1988, ApJ, 325, 49

Lawrence, C., et al., 1994, ApJL, 420, L9

Mao, S. \& Kochanek, C.S., 1994, MNRAS, 268, 569

Maoz, D., et al., 1993, ApJ, 409, 28

Maoz, D. \& Rix, H.-W., 1993, ApJ, 416, 425

Rix, H.-W., Maoz, D., Turner, E. \& Fukugita, M., 1994, ApJ, 435, 49

Turner, E., 1990, ApJL, 365, L43 